\title{
Suggestibility in functional neurological disorder: A meta-analysis
}

Lillian Wieder, BSc ${ }^{1}$, Richard J. Brown, $\mathrm{PhD}$, ClinPsyD ${ }^{2,3}$, Trevor Thompson, $\mathrm{PhD}^{4}, \&$ Devin B. Terhune, $\mathrm{PhD}^{1}$

${ }^{1}$ Department of Psychology, Goldsmiths, University of London, London, UK

${ }^{2}$ School of Health Sciences, University of Manchester, Manchester, UK

${ }^{3}$ Complex Trauma and Resilience Research Unit, Greater Manchester Mental Health NHS Foundation Trust, Manchester Academic Health Sciences Centre, Manchester, UK

${ }^{4}$ School of Human Sciences, University of Greenwich, London, UK

\section{Accepted for publication:}

22 September 2020

Journal of Neurology, Neurosurgery, and Psychiatry

\section{Corresponding author:}

Devin B. Terhune, PhD

Department of Psychology

Goldsmiths, University of London

8 Lewisham Way

New Cross, London, UK SE14 6NW

d.terhune@gold.ac.uk

Word count: 3,283 words 


\section{Abstract}

Objective Responsiveness to direct verbal suggestions (suggestibility) has long been hypothesized to represent a predisposing factor for functional neurological disorder (FND) but previous research has yielded conflicting results. The aim of this study was to quantitatively evaluate whether FND patients display elevated suggestibility relative to controls via meta-analysis.

Methods Four electronic databases were searched in November 2019, with the search updated in April 2020, for original studies assessing suggestibility using standardized behavioural scales or suggestive symptom induction protocols in FND (including somatization disorder) patients and controls. The metaanalysis followed Cochrane, PRISMA, and MOOSE guidelines. Data extraction and study quality coding were performed by two independent reviewers. Standardized suggestibility scores and responsiveness to symptom induction protocols were used to calculate standardized mean differences ( $M M D$ s) between groups. Results Of 26,643 search results, 19 articles presenting 11 standardized suggestibility datasets (FND: $n=316$; control: $n=360$ ) and 11 symptom suggestibility datasets (FND: $n=1285$; control: $n=1409$ ) were included in random-effects meta-analyses. Meta-analyses revealed that FND patients displayed greater suggestibility than controls on standardized behavioural scales (SMD, 0.48 [95\% CI, 0.15, 0.81]) and greater responsiveness to suggestive symptom induction ( $S M D, 1.39$ [95\% CI, 0.92, 1.86]). Moderation analyses presented mixed evidence regarding the extent to which effect sizes covaried with methodological differences across studies. No evidence of publication bias was found.

Conclusions These results corroborate the hypothesis that FND is characterized by heightened responsiveness to verbal suggestion. Atypical suggestibility may confer risk for FND and be a cognitive marker that can inform diagnosis and treatment of this condition. 


\section{Introduction}

Functional neurological disorder (FND) is characterized by impaired motor, cognitive and/or sensory functioning that resembles neurological pathology but is not adequately explained by, and is clinically distinguishable from, it. ${ }^{1,2}$ FND encompasses a diverse array of symptom subtypes including non-epileptic seizures, functional movement disorder, and somatization disorder, and is often conceptualized as a dissociative disorder. ${ }^{3}$ Previously referred to as hysteria, and also known as conversion disorder, functional neurological symptom disorder, and dissociative neurological symptom disorder, FND has a prevalence of 4-12 per $100,000^{4,5}$ and is found in $\sim 16 \%$ of neurology outpatients. ${ }^{6}$ It is associated with considerable diagnostic delays and frequent misdiagnosis, ${ }^{7}$ which add to the already significant psychological, social, and economic impact of the condition. ${ }^{8}$

FND has long been hypothesized to be characterized by elevated responsiveness to direct verbal suggestions (suggestibility) ${ }^{9}$ and suggestion and the expression of functional symptoms have been hypothesized to recruit overlapping mechanisms. ${ }^{10}$ Suggestibility is theorized to confer vulnerability for $\mathrm{FND}^{11}$ through aberrant meta-awareness of intentions, ${ }^{12-15}$ the capacity for suggestions to trigger automatized behavioural routines or mental representations, ${ }^{11}$ and/or a tendency to form precise (symptom) priors that override motor and perceptual systems. ${ }^{16,17}$ The use of suggestion to provoke FND symptoms is widely used to aid diagnosis ${ }^{18,19}$ and functional symptoms are responsive to suggestion-based treatments, such as hypnosis and placebo. ${ }^{20}$ Conditions with germane symptom profiles, such as dissociative disorders, are also characterized by elevated hypnotic suggestibility. ${ }^{21}$

Despite these various strands of evidence, the empirical association between suggestibility and FND seems to be highly variable. ${ }^{10,22}$ In order to quantify the evidence for atypical suggestibility in this population, we conducted a random-effects meta-analysis of controlled studies of suggestibility on standardized behavioural scales and in response to suggestive symptom induction protocols in all FND subtypes including somatization disorder/Briquet's syndrome. Secondary analyses investigated moderating influences on patient-control differences, including FND subtype, experimenter blindness, ${ }^{23}$ methodological quality, the inclusion of a hypnotic induction, ${ }^{24}$ and symptom provocation method. ${ }^{18}$ 


\section{Method}

\section{Eligibility criteria}

Inclusion criteria included (1) English language; (2) full paper in a peer-reviewed journal; (3) patient sample characterized by FND/symptoms, encompassing conversion disorder (DSM), dissociative neurological disorder (ICD), specific functional neurological syndromes (e.g., non-epileptic seizures), and conditions where functional neurological symptoms are a diagnostic feature (i.e. DSM-IV somatization disorder; Briquet's syndrome); (4) inclusion of a control group; and either (5) use of a standardized behavioural measure of direct verbal suggestibility ${ }^{24}$, or (6) assessment of symptom induction through suggestion (e.g., suggestive seizure induction ${ }^{18}$ ).

Exclusion criteria included (1) studies in which suggestion was used to aid diagnosis; (2) case studies/series or non-empirical papers; (3) overlapping/insufficient data; (4) use of interrogative suggestibility scales, which capture a different form of suggestibility characterized by high compliance ${ }^{25}$; and (5) studies of patients with functional somatic syndromes not specifically characterized by functional neurological symptoms (e.g., fibromyalgia).

\section{Search strategy}

PubMed, PsycINFO, Web of Science and Academic Search Complete databases were searched in November 2019 for eligible studies using terms relating to suggestibility and FND (see online supplemental content 1) and then integrated into a single database. The search was repeated in April 2020 but yielded no new studies. The reference lists of all eligible studies (and relevant review papers) were manually searched to identify additional studies. Authors were contacted when data were unavailable or to clarify ambiguities in methodology.

\section{Study selection}

Two raters (LW and a second rater) independently screened and assessed all studies for their eligibility using a two-stage procedure. First, all titles and abstracts were screened and articles not meeting eligibility criteria were rejected. Second, all remaining papers were reviewed to establish a final list of articles. Discrepancies at either stage were resolved in consultation with a third reviewer (DBT) and sometimes a fourth reviewer (RJB). Authors of eligible studies were contacted to address any questions regarding insufficient data. Of 5 
author groups contacted, $4(80 \%)$ provided data sufficient to permit study inclusion or answered queries that justified exclusion.

\section{Data extraction}

The two outcome types (standardized suggestibility and symptom suggestibility) were measured using continuous and categorical measures, respectively. After exclusion of two studies with overlapping data, data from eligible studies were extracted and coded independently by LW and the second rater using a data extraction form including: (i) study details (title, year, geographical location), (ii) diagnosis, (iii), diagnosis method, (iv) demographics (sample size and gender and age distributions), (v) study design details (suggestibility type [standardized or symptom], inclusion of a hypnotic induction, scale, administration method [live or recorded presentation], and scoring method [self or experimenter]), provocation method [see Table 2], symptom subtype, experimenter blindness [blind, unblind, or unreported]), and (vi) descriptive statistics ( $M \mathrm{~s}$ and $S D$ s [standardized suggestibility] or response counts [symptom suggestibility]). Symptom suggestibility response counts include only responses identified as typical for the respective patient (typicality reported: $k=8$; unreported: $k=3$ ). When studies reported results for more than one provocation method $(k=2)$, the rounded mean was used. Two studies included data for both standardized and symptom suggestibility. There was $91 \%$ agreement between the two raters and discrepancies were resolved with a third reviewer (DBT).

\section{Study quality}

A 13-item scale was developed to assess study quality (see online supplemental content 1). Items were adapted from an earlier meta-analysis that included items based on Cochrane criteria and PRISMA recommendations ${ }^{26}$ and a range of other methodological criteria such as experimenter blindness. LW and the second rater independently rated each item categorically $(0=$ criterion not met, $1=$ criterion met $)$ and a summed total was computed for each study. Agreement between raters was $90 \%(\mathrm{kappa}=.73)$ and discrepancies were resolved with DBT. 


\section{Meta-analysis and meta-regression}

Individual study effect sizes included between-group differences in suggestibility that were computed with standardised mean differences (SMDs; Hedges's gs) using Review Manager (RevMan v. 5.3, 2014; The Nordic Cochrane Centre, The Cochrane Collaboration, Copenhagen). Symptom suggestibility data consisted of binary outcomes that were used to compute odds ratios, which were subsequently transformed to $S M D \mathrm{~s}^{27}$ in MATLAB (v. 2017b, MathWorks, Natick, MA). SMDs were coded such that positive values reflected greater suggestibility in FND patients than controls. SMDs around 0.2, 0.5, and 0.8 are typically interpreted as reflecting small, moderate, and large effects, respectively.

Publication bias was assessed by examining funnel plots of effect sizes against standard errors for asymmetry, as might occur due to a small number of studies with small or non-significant effect sizes. We also tested for asymmetry using Egger's bias test ${ }^{28}$ where $p<.05$ is indicative of asymmetry and we report revised effect sizes correcting for asymmetry using the trim-and-fill method ${ }^{29}$ and funnel plots.

Random-effects meta-analyses were performed in JASP (v. 0.8.6, 2019; JASP Team, Netherlands). Outlier detection was made on the basis of studentized residuals $(>|3.3|) .{ }^{30}$ There were no outliers in the standardized suggestibility data (range: -1.63 to 1.33 ) or the symptom suggestibility data (range: -2.01 to 1.26). Moderating effects were assessed using meta-regression analyses whenever data were available for at least 2 studies at each level of a categorical moderator and at least 10 studies for continuous moderators. ${ }^{31}$ Moderators included five categorical measures (symptom subtype, experimenter blindness, hypnotic induction, symptom provocation method [suggestion vs. nocebo; symptom studies], symptom typicality [not reported vs. report; symptom studies]; and control type [non-clinical vs. clinical; standardized studies]) and one continuous measure (methodological quality).

\section{Results}

\section{Study inclusion}

A PRISMA diagram showing study selection is presented in Figure 1. Principal features of these studies, including diagnosis criteria and procedures, are presented in Tables 1 and $\mathbf{2 .}$ 


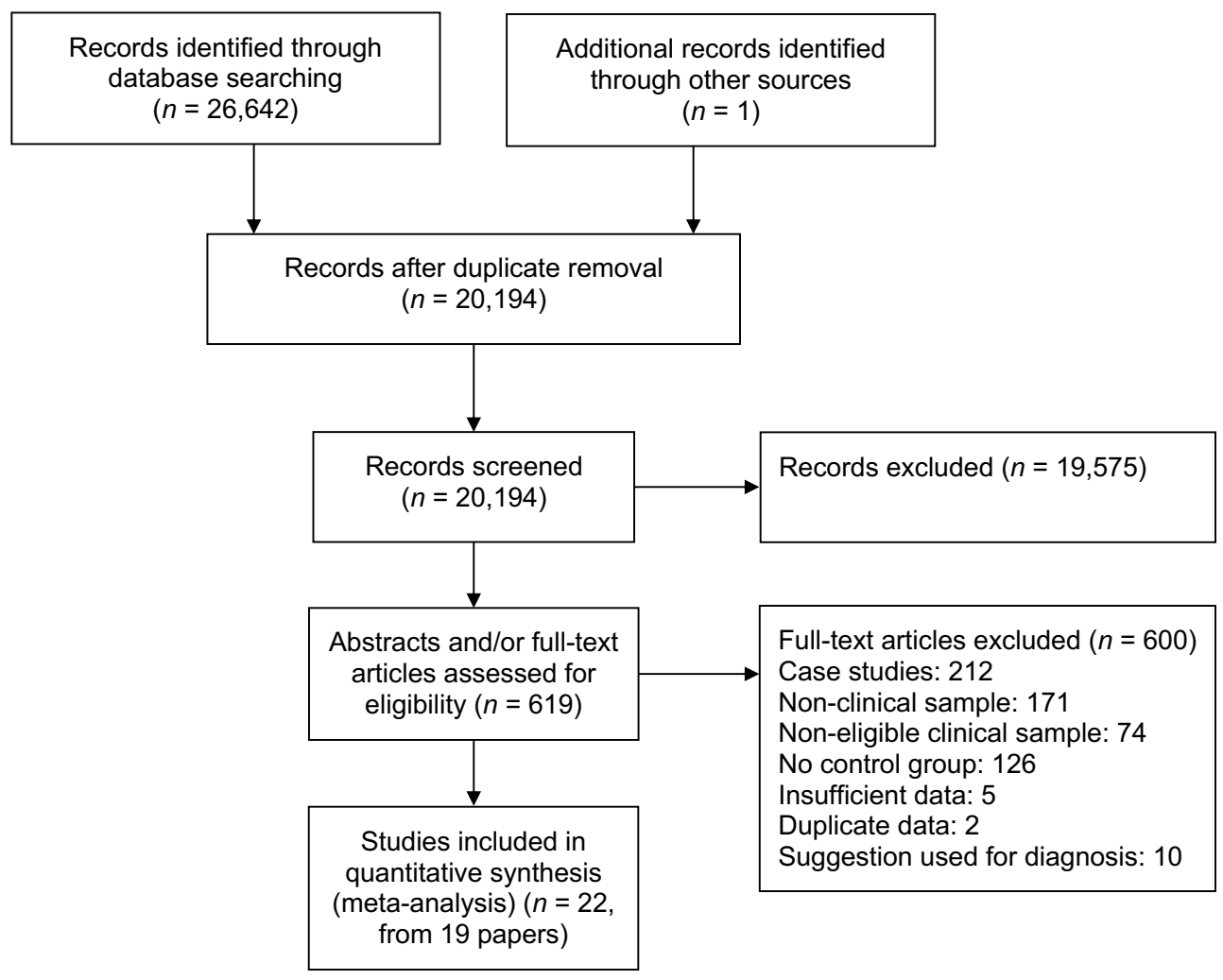

Figure 1. PRISMA flowchart of study selection process.

Table 1. Characteristics of included studies measuring standardized suggestibility in FND

\begin{tabular}{|c|c|c|c|c|c|c|c|c|c|c|}
\hline \multirow[t]{2}{*}{ Source } & \multicolumn{2}{|c|}{ Diagnostic criteria } & \multicolumn{3}{|c|}{ FND } & \multicolumn{3}{|c|}{ Controls } & \multirow[t]{2}{*}{ Scale } & \multirow{2}{*}{$\begin{array}{l}\text { Hypnotic } \\
\text { induction }\end{array}$} \\
\hline & Formal & Procedure & Diagnosis & $\begin{array}{c}n \\
(\% \\
\text { female) }\end{array}$ & $\begin{array}{c}\text { Age } \\
{[M(\mathrm{SD})]}\end{array}$ & Diagnosis & $\begin{array}{c}n \\
(\% \\
\text { female) }\end{array}$ & $\begin{array}{c}\text { Age } \\
{[M(\mathrm{SD})]}\end{array}$ & & \\
\hline Khan et al., 2009 & - & MI & NES & $24(-)$ & $-(-)$ & ES & $16(-)$ & $-(-)$ & HIP & $\mathrm{Y}$ \\
\hline Brown et al., 2008 & $\begin{array}{l}\text { DSM- } \\
\text { IV }\end{array}$ & $\mathrm{CR}$ & SD & 19 (95) & $40.9(14.1)$ & DYS & $17(59)$ & $45.4(11.1)$ & BSS & $\mathrm{N}$ \\
\hline Roelofs et al., 2002 & $\begin{array}{l}\text { DSM- } \\
\text { IV }\end{array}$ & MI & $\mathrm{mCD}$ & $50(84)$ & $37.2(11.9)$ & $\mathrm{mAD}$ & $50(82)$ & $36.4(11.1)$ & SHSS:C & $\mathrm{Y}$ \\
\hline $\begin{array}{l}\text { Litwin and Cardeña, } \\
2001\end{array}$ & - & MI & NES & $10(100)$ & 30.5 (9.9) & ES & $31(45)$ & $35.2(8.9)$ & SHCS & $\mathrm{Y}$ \\
\hline $\begin{array}{l}\text { Moene et al., 2001 / } \\
\text { Spinhoven et al. } \\
\text { 1991* }\end{array}$ & $\begin{array}{l}\text { DSM- } \\
\text { III-R }\end{array}$ & $\mathrm{CR}$ & $\mathrm{mCD}$ & $96(-)$ & $-(-)$ & $\mathrm{NCC}$ & $82(57)$ & $40.1(14.3)$ & SHCS & $\mathrm{Y}$ \\
\hline Barry et al., 2000 & - & $\mathrm{MI}, \mathrm{CR}$ & NES/ES & $36(-)$ & $-(-)$ & ES & $22(-)$ & $-(-)$ & HIP & $\mathrm{Y}$ \\
\hline $\begin{array}{l}\text { Goldstein et al., } \\
2000\end{array}$ & - & $\mathrm{MI}, \mathrm{CR}$ & NES & $20(80)$ & 34.4 (12.4) & $\mathrm{NCC}$ & $20(45)$ & $36(8.5)$ & CIS & $\mathrm{N}$ \\
\hline Kuyk et al., 1999 & - & MI & NES & $20(80)$ & $25(-)$ & ES & $17(18)$ & $37(-)$ & SHCS & $\mathrm{Y}$ \\
\hline Kuyk et al., 1995 & - & $\mathrm{MI}, \mathrm{CR}$ & NES & $6(50)$ & 19.3 & ES & $7(14)$ & $28.6(-)$ & SHCS & $\mathrm{Y}$ \\
\hline Bliss, 1984a & - & $\mathrm{CR}$ & $\mathrm{mCD}$ & $18(100)$ & $-(-)$ & $\mathrm{NCC}$ & $49(-)$ & $-(-)$ & SHSS:C & $\mathrm{Y}$ \\
\hline Bliss, $1984 \mathrm{~b}$ & ـ & $\mathrm{CR}$ & BS & $17(100)$ & $-(-)$ & $\mathrm{NCC}$ & $49(-)$ & $-(-)$ & SHSS:C & $\mathrm{Y}$ \\
\hline
\end{tabular}

Notes. BSS = Barber Suggestibility Scale; BS=Briquet's syndrome; CIS = Creative Imagination Scale; CR=case note review for ruling out organic causes; DSM=Diagnostic and Statistical Manual of Mental Disorders (American Psychiatric Association); DYS=dystonia; ES=epileptic seizures; FND=functional neurological disorder; HIP = Hypnotic Induction Profile; $\mathrm{mAD}=$ mixed affective disorders; mixed conversion disorder (mCD); $\mathrm{MI}=$ Medical investigations; $\mathrm{NCC}=$ non-clinical controls; NES=non-epileptic seizures; $\mathrm{PDF}=$ positive diagnostic features; $\mathrm{SD}=$ somatization disorder; SHCS = Stanford Hypnotic Clinical Scale; $\mathrm{SHSS}: \mathrm{C}=$ Stanford Hypnotic Susceptibility Scale: Form C; - = Not reported; * = Control data were drawn from Spinhoven et al. (1991). 
Table 2. Characteristics of included studies measuring symptom suggestibility in FND

\begin{tabular}{|c|c|c|c|c|c|c|c|c|c|c|c|c|}
\hline \multirow[t]{2}{*}{ Source } & \multicolumn{2}{|c|}{ Diagnostic criteria } & \multicolumn{3}{|c|}{ FND } & \multicolumn{2}{|c|}{ Controls } & \multirow[b]{2}{*}{$\begin{array}{c}\text { Age } \\
{[M(\mathrm{SD})]}\end{array}$} & \multicolumn{2}{|c|}{ Method } & \multicolumn{2}{|c|}{ Responsiveness: $n(\%)$} \\
\hline & Formal & Procedure & Diagnosis & $\begin{array}{c}n(\% \\
\text { female })\end{array}$ & $\begin{array}{c}\text { Age } \\
{[M(\mathrm{SD})]}\end{array}$ & Diagnosis & $\begin{array}{c}n(\% \\
\text { female })\end{array}$ & & $\begin{array}{c}\text { Suggestion/noceb } \\
0\end{array}$ & $\begin{array}{l}\text { Hypnotic } \\
\text { induction }\end{array}$ & FND & Control \\
\hline $\begin{array}{l}\text { Kandler et al., } \\
2018\end{array}$ & - & MI, CR & NES & $416(-)$ & $-(-)$ & ES & $568(-)$ & $-(-)$ & VS, HV, PS & $\mathrm{N}$ & $\begin{array}{c}120 \\
(29 \%)\end{array}$ & $40(7 \%)$ \\
\hline $\begin{array}{l}\text { Chen-Block et al., } \\
2016\end{array}$ & - & MI, CR & NES/ES & $\begin{array}{l}466 \\
(70)\end{array}$ & $-(-)$ & ES & $\begin{array}{l}581 \\
(54)\end{array}$ & $-(-)$ & $\begin{array}{c}\text { VS, HV, PS, AP, } \\
\text { ST }\end{array}$ & $\mathrm{N}$ & $\begin{array}{c}240 \\
(52 \%)\end{array}$ & $15(3 \%)$ \\
\hline Goyal et al., 2014 & - & MI & NES & $\begin{array}{l}140 \\
(71)\end{array}$ & $\begin{array}{l}21.38 \\
(10.83)\end{array}$ & ES & $50(34)$ & $\begin{array}{l}21.18 \\
(9.98)\end{array}$ & $\begin{array}{l}\text { VS, PS, VTF, } \\
\text { TC, MS, SI }\end{array}$ & $\mathrm{N}$ & $84(60 \%)$ & $0(0 \%)$ \\
\hline Arain et al., 2009 & - & MI & NES & $24(67)$ & $-(-)$ & ES & $54(65)$ & $-(-)$ & VS, HV & $\mathrm{N}$ & $8(33 \%)$ & $6(11 \%)$ \\
\hline Khan et al., 2009 & - & MI & NES & $24(-)$ & $-(-)$ & ES & $16(-)$ & $-(-)$ & VS & $\mathrm{Y}$ & $11(46 \%)$ & $2(13 \%)$ \\
\hline $\begin{array}{l}\text { Kenney et al., } \\
2007\end{array}$ & FW & PDF & PT & $12(-)$ & $42.5(11)$ & ET & $33(-)$ & $56.8(17)$ & VS, HV, VTF & $\mathrm{N}$ & $6(50 \%)$ & $5(15 \%)$ \\
\hline $\begin{array}{l}\text { Martinez-Taboas, } \\
2002\end{array}$ & - & $\mathrm{CR}, \mathrm{PDF}$ & NES & $8(88)$ & $32(9.2)$ & ES & $5(100)$ & $30(6.2)$ & VS, ARS & $\mathrm{Y}$ & $8(100 \%)$ & $0(0 \%)$ \\
\hline Barry et al., 2000 & - & MI, CR & NES/ES & $47(-)$ & $-(-)$ & ES & $22(-)$ & $-(-)$ & VS & $\mathrm{Y}$ & $36(77 \%)$ & $1(5 \%)$ \\
\hline $\begin{array}{l}\text { Chabrol et al, } \\
1995\end{array}$ & $\begin{array}{l}\text { DSM- } \\
\text { IV }\end{array}$ & CR, PDF & $\mathrm{mCD}$ & $15(-)$ & $44(14)$ & $\mathrm{mND}$ & $40(-)$ & $53(17)$ & VS & $\mathrm{N}$ & $6(40 \%)$ & $17(43 \%)$ \\
\hline $\begin{array}{l}\text { Lancman et al., } \\
1994\end{array}$ & - & MI & NES & $93(77)$ & $26.7(-)$ & ES & $20(70)$ & $28.2(-)$ & $\mathrm{VS}, \mathrm{AP}$ & $\mathrm{N}$ & $72(77 \%)$ & $0(0 \%)$ \\
\hline $\begin{array}{l}\text { Walczak et al., } \\
1994\end{array}$ & & MI & NES & $40(90)$ & $33.9(11.6)$ & ES & $20(45)$ & $34(11.7)$ & VS, SI & $\mathrm{N}$ & $33(83 \%)$ & $5(25 \%)$ \\
\hline
\end{tabular}

Notes. $\mathrm{AP}=$ alcohol patch; $\mathrm{ARS}=$ age regression suggestion; $\mathrm{CR}=$ case note review for ruling out organic causes; $\mathrm{DSM}=$ Diagnostic and Statistical Manual of Mental Disorders (American Psychiatric Association); ES=epileptic seizures; ET=essential tremor; $\mathrm{FW}=$ Fahn and Williams diagnostic criteria; $\mathrm{HV}=$ hyperventilation; $\mathrm{MI}=$ Medical investigations; $\mathrm{mCD}=$ mixed conversion disorder $(\mathrm{mCD}) ; \mathrm{mND}=$ mixed neurological disorders; $\mathrm{MS}=$ moist swab; $\mathrm{NES}=$ non-epileptic seizures; $\mathrm{PDF}=$ positive diagnostic features; $\mathrm{PS}=$ photic stimulation; $\mathrm{PT}=$ psychogenic tremor; $\mathrm{SI}=$ saline injection; $\mathrm{ST}=$ subjective trigger; $\mathrm{TC}=$ temple compression; $\mathrm{VS}=$ verbal suggestion; VTF=vibrating tuning fork; - = Not reported.

\section{Study and participant characteristics}

The 19 papers included 11 standardized suggestibility studies (FND: $n=316$, control: $n=360$ ) and 11 symptom suggestibility studies (FND: $n=1285$, control: $n=1409$ ) (see online supplemental content 1 for references). FND patients primarily comprised those with non-epileptic seizures ( $k=6$ [standardized]; $k=9$ [symptom]), with small numbers of studies of patients with mixed conversion disorder ( $k=3$ [standardized] and $k=1$ [symptom]), somatization disorder/Briquet's syndrome ( $k=2$ [standardized]), and psychogenic tremor $(k=1$ [symptom]). The standardized studies were published between 1984 and 2009 and were conducted in the US $(k=5)$, the Netherlands $(k=4)$, and the UK $(k=2)$. The symptom studies were published between 1994 and 2018, and were conducted in the US $(k=7)$, Puerto Rico $(k=1)$, France $(k=1)$, India $(k=1)$ and the UK $(k=1)$. The mean percentage of females for standardized studies was 86.13 for FND $(k=8)$ and 45.71 for controls $(k=7)$, whereas for symptom studies $(k=6)$ it was 77.17 for FND and 61.33 for controls. In the standardized studies, the mean age for FND was $31.22(S D=8.01)(k=6)$, and for controls it was 36.96 $(S D=5.09)(k=7)$, whereas for the symptom studies $(k=6)$, mean age for FND was $33.41(S D=8.79)$, and $37.20(S D=14.38)$ for controls. 
Details of the types of standardized scales and provocation methods as well as the use of a hypnotic induction are provided in Table 1 and Table 2, respectively. A hypnotic induction was used in 9 standardized studies and 3 symptom studies. All symptom studies included verbal suggestion but varied in their use of various nocebo procedures.

\section{Methodological quality criteria}

Ratings for each study on each of the methodological quality criteria items are shown in Supplementary

Tables 1 and 2. Although some of the criteria were met by the majority of studies, multiple criteria were not reliably met. Only 5 of 22 studies (23\%) reported that the experimenter was blind to group, $18(82 \%)$ described the inclusion/exclusion criteria, $16(73 \%)$ described the diagnosis procedure and criteria in adequate detail, $10(45 \%)$ described participant characteristics and $3(14 \%)$ exhibited demographic comparability between patients and controls. In the standardized studies, 7 of $11(64 \%)$ described the scale and procedure in adequate detail, and only $1(9 \%)$ included a measure to correct for compliance. In the symptom studies, 8 of $11(73 \%)$ clearly described the provocation method.

\section{Meta-analysis of standardized suggestibility}

Meta-analysis of 11 standardized behavioural suggestibility studies found that patients with an FND exhibited greater suggestibility than controls, $S M D=0.48[0.15,0.81], Z=2.84, p=.004$ (see Figure 2). Positive results were observed in 8 studies with a high inconsistency of effect sizes across studies, $I^{2}=73 \%$. Jackknife analysis in which each study effect was sequentially omitted and the analysis re-performed indicated that the group difference was reliably significant (SMD range: $0.40-0.56$ ). 


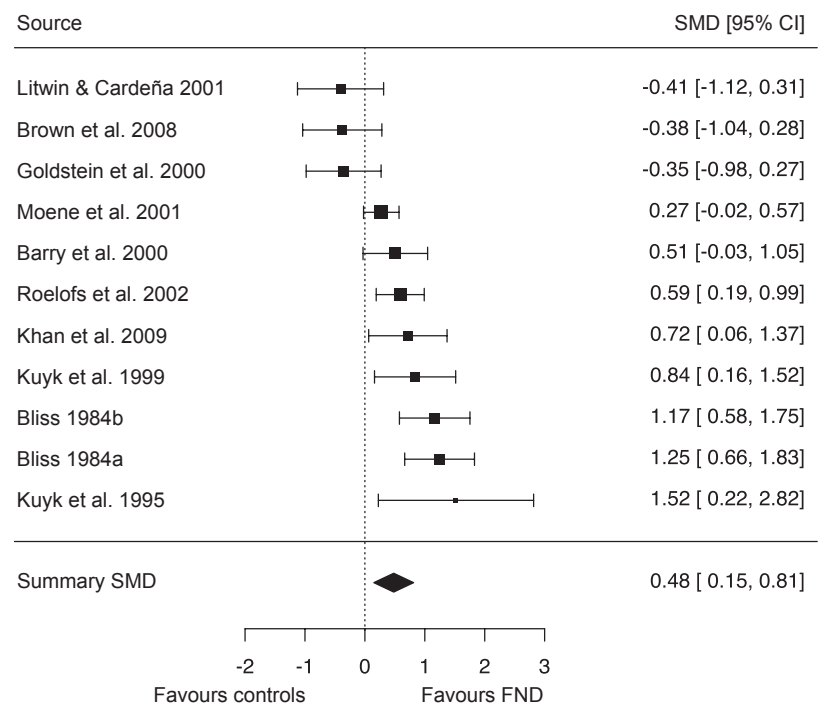

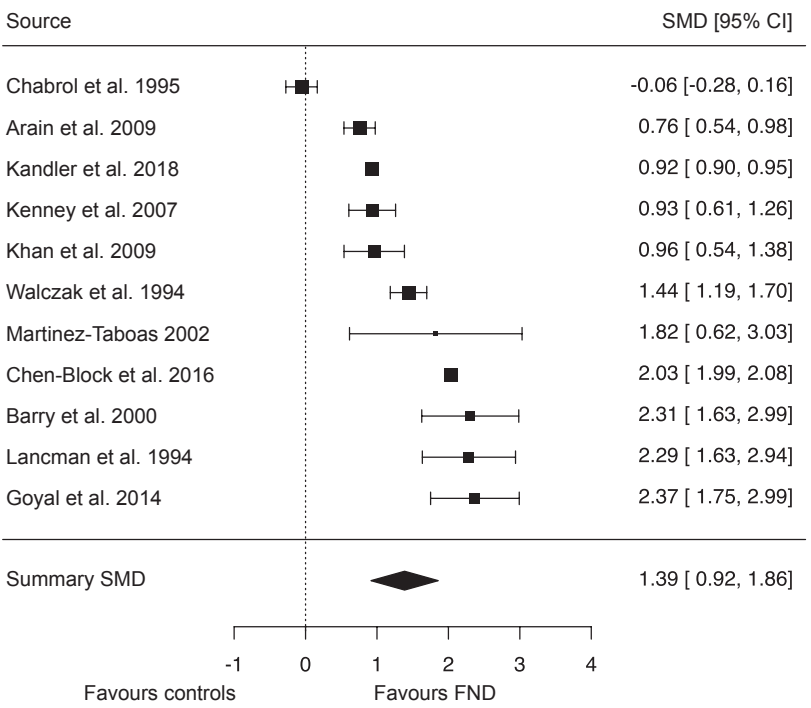

Figure 2. Forest plots of Standardised Mean Differences (SMDs) (with 95\% confidence intervals) from (left) 11 standardized suggestibility studies and (right) 11 symptom suggestibility studies. Marker sizes reflect study weights with smaller markers denoting smaller weights.

\section{Meta-analysis of symptom suggestibility}

Meta-analysis of 11 symptom suggestibility studies found that patients with FND displayed greater responsiveness than controls, $S M D=1.39[0.92,1.86], \mathrm{z}=5.77, p<.001$ (see Figure 2). Overall positive responses were observed in $49 \%$ of patients with FND and $6 \%$ of controls (sensitivity $=49 \%$, specificity=94\%). Ten of the 11 studies exhibited positive $S M D$ s although there was substantial heterogeneity in effect sizes across studies, $I^{2}=99 \%$. Jackknife analysis revealed that the group difference remained significant after omitting each study in a sequential manner (SMD range: 1.25 to 1.54$)$.

\section{Meta-analysis of standardized vs. symptom suggestibility}

The weighted effect size for symptom studies was significantly greater than that for standardized studies, $z=2.60, p=.009$. This difference remained stable, $z=2.29, p=.022$, after removing the two studies included in both data sets. When the two data sets were aggregated, the cumulative standardised effect size was slightly less than $1, S M D=0.96[0.62,1.29]$. 


\section{Publication bias}

Egger's test did not suggest asymmetry in the distribution of effect sizes in the standardized studies, $z=0.69$, $p=.49$, or in the symptom studies, $z=1.50, p=.13$. A trim-and-fill estimate produced only a slight reduction in effect sizes for standardized studies, $\triangle S M D=-.05$, and symptom studies, $\triangle S M D=-.10$ (see Figure 3).
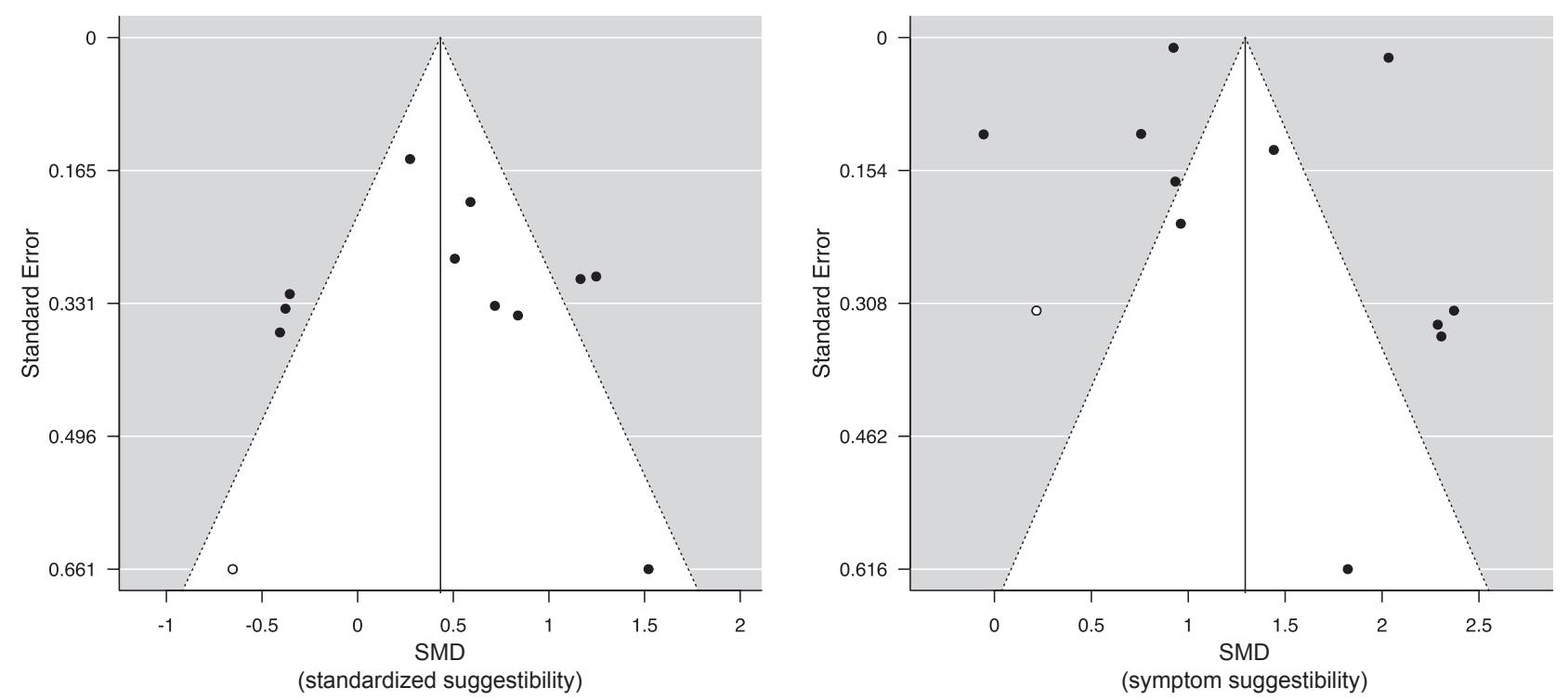

Figure 3. Funnel plots of standardized mean differences (SMDs) from (left) 11 standardised suggestibility studies and (right) 11 symptom suggestibility studies. Filled circles denote individual study effect sizes and empty circles denote estimated missing individual effect sizes due to publication bias imputed using the trim-and-fill method. Summary $S M D$ s $[95 \% \mathrm{CI}]$ using the trim-and-fill method were $S M D=0.43[0.10,0.76]$ (standardized suggestibility studies) and $S M D=1.29[0.84,1.75]$ (symptom suggestibility studies).

\section{Meta-regression of standardized and symptom suggestibility}

Given the observed inconsistency in the magnitude of effects, a set of meta-regression analyses considered whether group differences were moderated by binary and continuous predictors pertaining to symptom subgroups and study methodologies (see Table 3). Non-epileptic seizure (NES) patients exhibited marginally significantly larger effect sizes than mixed-FND patients in the symptom studies although this difference was driven by a single non-significant mixed-FND study. These symptom subgroups did not significantly differ in the standardized studies. Effect sizes were significantly larger when a hypnotic induction was included in standardized studies, but not in symptom studies. Effect sizes were also larger in studies that reported whether suggested symptoms were typical for the patient relative to those that did not 
report this information; this implies that effect sizes are not inflated by the inclusion of atypical symptoms in response rates for suggestive symptom induction. By contrast, effect sizes were not significantly related to the type of control (clinical vs. non-clinical; standardized studies) or suggestive induction protocol (suggestion vs. nocebo [suggestion and sham]; symptom studies). Experimenter blindness did not significantly moderate group differences with numerical differences in opposing directions for standardized and symptom studies. Similarly, methodological quality related to effect sizes in opposing directions: greater quality was significantly associated with lower effect sizes in standardized studies, but with larger, albeit non-significantly, effect sizes in symptom studies. These effects were primarily driven by procedure description. Standardized effect sizes were smaller in studies that met criteria for clarity of inclusion/exclusion criteria: $z=-2.54, p=.011$; diagnostic procedure: $z=-2.82, p=.005$; and scale administration procedure: $z=-2.28, p=.022$ (all other $p \mathrm{~s}>.09$ ). In contrast, symptom effect sizes were larger in studies that met criteria for clarity of diagnostic procedure, $z=3.23, p=.001$ (all other $p \mathrm{~s}>.09$ ).

Table 3. Meta-regression analyses for standardized and symptom suggestibility studies (SMD [95\% CIs] $(k))$

\begin{tabular}{|c|c|c|c|c|c|}
\hline \multirow{2}{*}{$\begin{array}{l}\text { Moderator } \\
\text { Suggestibility type }\end{array}$} & \multicolumn{2}{|c|}{ Analysis groups } & \multirow[t]{2}{*}{$\boldsymbol{Z}$} & \multirow[t]{2}{*}{$p$} & \multirow[t]{2}{*}{$I^{2}$} \\
\hline & Mixed-FND & NES & & & \\
\hline Standardized & $0.58[0.10,1.06](5)$ & $0.39[-0.12,0.90(6)$ & -0.54 & .59 & $75 \%$ \\
\hline Symptom & $0.43[-0.54,1.40](2)$ & $1.62[1.09,2.14](9)$ & 1.96 & .050 & $99 \%$ \\
\hline Hypnotic induction & No & Yes & & & \\
\hline Standardized & $-0.37[-0.82,0.09](2)$ & $0.66[0.34,0.97](9)$ & 2.74 & .006 & $61 \%$ \\
\hline Symptom & $1.30[0.76,1.85](8)$ & $1.66[0.67,2.64])(3)$ & 0.62 & .54 & $100 \%$ \\
\hline Experimenter blindness & Unblind & Blind & & & \\
\hline Standardized & $0.46[0.03,0.89](7)$ & $0.54[-0.09,1.16](4)$ & 0.20 & .84 & $76 \%$ \\
\hline Symptom & $1.44[0.94,1.93](10)$ & $0.93[0.61,1.26](1)$ & & & \\
\hline $\begin{array}{l}\text { Suggestive symptom } \\
\text { induction method }\end{array}$ & Suggestion & Nocebo & & & \\
\hline Symptom & $1.20[0.11,2.28](4)$ & $1.50[0.93,2.08](7)$ & 0.69 & .49 & $100 \%$ \\
\hline $\begin{array}{l}\text { Suggestive symptom } \\
\text { typicality }\end{array}$ & Not reported & Typical & & & \\
\hline Symptom & $0.60[-0.14,1.34](3)$ & $1.70[1.14,2.26](8)$ & 2.07 & .038 & $100 \%$ \\
\hline Control sample type & Non-clinical & Clinical & & & \\
\hline Standardized & $0.58[-0.08,1.24](4)$ & $0.42[0.01,0.82](7)$ & -0.40 & .69 & $76 \%$ \\
\hline Methodologica & & & & & \\
\hline Standardized & $12-$ & cale & -2.21 & .027 & $66 \%$ \\
\hline Symptom & 10-ite & scale & 1.76 & .079 & $99 \%$ \\
\hline
\end{tabular}

Notes. FND=functional neurological disorder; NES=non-epileptic seizures 


\section{Discussion}

The results of this meta-analysis suggest that FND patients display elevated suggestibility on standardized behavioural scales and in response to suggestive symptom induction protocols, consistent with theoretical predictions to this effect. ${ }^{9,10,22}$ There was no evidence for publication bias although there was considerable heterogeneity in both data sets, which was partly explained by methodological variability.

These findings are consistent with models proposing responsiveness to suggestion as a vulnerability factor for FND and those attributing functional symptoms to precise symptom priors. ${ }^{11,16}$ Recent theoretical work conceptualizes functional symptoms as arising from the automatic activation of "rogue" mental representations or symptom priors akin to Janet's fixed ideas. Numerous factors have been proposed to moderate this process including autonomic arousal, inhibitory deficits, and problems with body perception. ${ }^{11}$ Suggestibility may confer heightened sensitivity to symptom-specific cues or dissociative responses to stressors. ${ }^{32}$ Moreover, suggestibility has been proposed to reflect a generalized tendency to form precise priors that override motor and perceptual systems,${ }^{33}$ which is thought to be a key process in $\mathrm{FND}^{16}$ and symptom reporting more generally. ${ }^{17}$ This aligns with research showing that hypnotic suggestibility predicts symptom severity in FND patients. ${ }^{34}$ Observed links between hypnotic suggestibility and emotional responsiveness to social cues,${ }^{24}$ highlight the potential role of suggestibility in symptom modelling or triggering through social observation. ${ }^{11}$ The perception that functional symptoms are extra-volitional may be further augmented by aberrant meta-awareness of intentions, as observed in both $\mathrm{FND}^{14,15}$ and high hypnotic suggestibility. ${ }^{12,13}$

Although the two forms of suggestibility moderately covary, ${ }^{35}$ FND patients were more responsive to symptom-specific $(S M D=1.39[0.92,1.86])$ than to standardized suggestions $(S M D=0.48[0.15,0.81])$, implying selectively greater suggestibility for functional symptoms. Indeed, the sole non-significant provocation study ${ }^{36}$ administered suggestions for generic symptoms that did not necessarily mirror patients' symptom profiles. Previous research similarly found that highly dissociative individuals and dissociative and acute stress disorder patients are more responsive to suggestions for dissociative experiences (e.g., amnesia). ${ }^{21}$ Symptom suggestibility in FND may thus partly reflect a kindling process whereby symptoms become more responsive to verbal suggestion over time. ${ }^{11,37}$

Atypical suggestibility in FND patients complements research showing greater suggestibility in germane conditions, such as dissociative and stress disorders ${ }^{10,21}$ and somatoform disorders. ${ }^{38}$ The overlapping 
symptom profiles of these conditions implies an association between suggestibility and dissociative psychopathology, the specificity of which is corroborated by research indicating that suggestibility does not seem to be a characteristic of general psychopathology. ${ }^{39,40}$ Variability in detachment and compartmentalization symptoms ${ }^{41}$ and exposure to stressful life events may account for heterogeneity in the suggestibility profiles of FND patients, with heightened responsiveness to suggestions being specific to, or more pronounced among, patients with marked dissociative symptomatology. ${ }^{34,42}$ Further research is required to assess whether this heterogeneity is attributable to the presence of a highly suggestible subtype among FND patients, who may be particularly likely to benefit from suggestion-based interventions. An additional outstanding question is whether atypical suggestibility is a feature shared across different FND symptom subtypes. The available evidence suggests this is the case: NES patients were not reliably more suggestible than other FND patients and independent research has documented or implied elevated responsiveness to suggestion in psychogenic parkinsonism, ${ }^{43}$ functional movement disorders, ${ }^{44-47}$ and functional vision loss. ${ }^{48}$ Moderation analyses indicated that the administration of a hypnotic induction was associated with greater standardized suggestibility among FND patients. This is consistent with the proposal that individuals with compartmentalization symptoms benefit more from a hypnotic induction ${ }^{21}$ although the mechanistic basis of this effect remains unclear. ${ }^{24}$

These effects attest to the efficacy of suggestion in the diagnosis of FND. ${ }^{18}$ Suggestive symptom induction displayed high specificity (94\%) although sensitivity is poor (49\%), indicating that this technique is insufficient as a standalone diagnostic procedure. The inclusion of sham methods or a hypnotic induction were not associated with greater discrimination of FND patients and controls relative to verbal suggestion alone but warrant further attention. Suggestive symptom induction is likely to be especially valuable in suggestible populations such as adolescent and elderly samples ${ }^{24}$ or patients with comorbid dissociative or stress disorder diagnoses. ${ }^{10,21}$ It may also inform diagnosis of comorbid non-epileptic seizures in epilepsy patients $^{49}$ and medication prescription ${ }^{50}$ and prognosis $^{51}$ in FND patients. Insofar as suggestibility is a positive predictor of outcome with suggestion-based treatments, ${ }^{52}$ these results also support greater incorporation of suggestion techniques in treatment protocols, which show promising results in randomizedcontrolled trials. ${ }^{20}$ However, they also highlight the need to control for suggestion, or consider its role, in diagnostic and treatment procedures, particularly those that evoke strong response expectancies. ${ }^{46}$ 


\section{Limitations}

The principal limitations of these data concern methodological variability across studies. Methodological quality was significantly or descriptively related to effect sizes in both data sets albeit in opposite directions. Among standardized studies, older studies that did not precisely specify inclusion/exclusion criteria and/or diagnostic and scale administration procedures tended to exhibit larger effect sizes; in contrast, precise specification of diagnostic procedures was associated with larger effect sizes in symptom studies. In most studies, the operator administering the assessment was not blind to patient group, which may inflate effect $\operatorname{sizes}^{23}$, although there was no evidence for an experimenter effect in the standardized studies. The sole blind symptom study had a lower effect size than the remainder of the studies but was still large in magnitude $(S M D=0.93[0.61,1.26])$. Most studies included clinical controls (e.g., epilepsy patients), which raises issues regarding generalizability although effect sizes did not significantly relate to control type. Similarly, the majority of FND samples comprised patients presenting with non-epileptic seizures and thus further controlled research is required to assess responsiveness to suggestion across a range of FND symptom subtypes, particularly those presenting with cognitive symptoms. Symptom suggestibility estimates are also confounded by baseline symptom frequency, which is not incorporated into these assessments. ${ }^{18}$ This potentially renders patients with high symptom frequency at an increased risk of false positive responses, although there is evidence that this is not the case. ${ }^{53}$ The studies also varied in whether successful responses to symptom induction protocols were contingent upon the typicality of the response, which accounted for variability in effect sizes. Standardized studies were limited insofar as only one controlled for compliance. In addition, standardized suggestibility scales include a disproportionate number of suggestions for dissociative and functional symptoms (e.g., paralysis), ${ }^{10,24,34}$ raising the question of whether elevated suggestibility in FND generalizes beyond these symptoms. Collectively, these findings underscore the need for optimization and standardization of suggestive symptom induction protocols, ${ }^{18}$ compliance-correction, ${ }^{24}$ and more diverse suggestion batteries.

\section{Conclusions}

This meta-analysis corroborates the long-held view that FND is characterized by elevated suggestibility. Increased suggestibility has direct implications for the risk factors underlying this condition, the use of 
suggestion to aid diagnosis, the utility of suggestion-based treatments for functional symptoms, and heterogeneity within this population.

\section{Acknowledgements}

The authors thank Che Ofuasia, Goldsmiths, University of London, for contributing to the data coding and evaluation.

\section{Contributors}

All authors conceived the project. LW carried out the database searches and data coding with assistance from RJB, TT, and DBT. LW and DBT performed the meta-analysis with assistance from RJB and TT. LW and DBT drafted the initial manuscript. All authors reviewed and approved the final version of the manuscript.

\section{Funding}

This study was supported by Bial Foundation bursary 70/16 (DBT) and a Gyllenbergs Foundation fellowship [no number] (DBT).

\section{Competing interests}

No, there are no competing interests for any author

\section{Patient consent for publication}

Not required.

\section{Provenance and peer review}

Not commissioned; externally peer reviewed. 


\section{References}

1. Espay AJ, Aybek S, Carson A, et al. Current concepts in diagnosis and treatment of functional neurological disorders. JAMA Neurol 2018;75:1132-1141.

2. American Psychiatric Association. Diagnostic and statistical manual of the mental disorders (5th Ed.). Arlington, VA: American Psychiatric Press, 2013.

3. Brown RJ. Dissociation and functional neurologic disorders. Handb Clin Neurol 2016;139:85-94.

4. Carson AJ, Brown R, David AS, et al. Functional (conversion) neurological symptoms: Research since the millennium. J Neurol Neurosurg Psychiatry 2012;83:842-850.

5. Benbadis SR, Allen Hauser W. An estimate of the prevalence of psychogenic non-epileptic seizures. Seizure 2000;9:280-281.

6. Stone J, Carson A, Duncan R, et al. Who is referred to neurology clinics? The diagnoses made in 3781 new patients. Clin Neurol Neurosurg 2010;112:747-751.

7. Reuber M. The etiology of psychogenic non-epileptic seizures: Toward a biopsychosocial model. Neurol Clin 2009;27:909-924.

8. Gelauff J, Stone J, Edwards M, Carson A. The prognosis of functional (psychogenic) motor symptoms: a systematic review. J Neurol Neurosurg Psychiatry 2014;85:220-226.

9. Janet P. The major symptoms of hysteria. London, UK: Macmillian \& Co., 1907.

10. Bell V, Oakley DA, Halligan PW, Deeley Q. Dissociation in hysteria and hypnosis: Evidence from cognitive neuroscience. Journal of Neurology, Neurosurgery, and Psychiatry 2011;82:332-339.

11. Brown RJ, Reuber M. Towards an integrative theory of psychogenic non-epileptic seizures (PNES). Clin Psychol Rev 2016;47:55-70.

12. Lush P, Naish P, Dienes Z. Metacognition of intentions in mindfulness and hypnosis. Neuroscience of Consciousness 2016:1-10.

13. Terhune DB, Hedman LRA. Metacognition of agency is reduced in high hypnotic suggestibility. Cognition 2017;168:176-181.

14. Baek K, Donamayor N, Morris LS, et al. Impaired awareness of motor intention in functional neurological disorder: Implications for voluntary and functional movement. Psychol Med 2017;47:16241636. 
15. Jungilligens J, Wellmer J, Schlegel U, Kessler H, Axmacher N, Popkirov S. Impaired emotional and behavioural awareness and control in patients with dissociative seizures. Psychol Med 2019:1-9.

16. Edwards MJ, Adams RA, Brown H, Parees I, Friston KJ. A Bayesian account of 'hysteria'. Brain 2012;135:3495-3512.

17. Van den Bergh O, Witthoft M, Petersen S, Brown RJ. Symptoms and the body: Taking the inferential leap. Neurosci Biobehav Rev 2017;74:185-203.

18. Popkirov S, Gronheit W, Wellmer J. A systematic review of suggestive seizure induction for the diagnosis of psychogenic nonepileptic seizures. Seizure 2015;31:124-132.

19. LaFaver K, Lang AE, Stone J, et al. Opinions and clinical practices related to diagnosing and managing functional (psychogenic) movement disorders: changes in the last decade. Eur J Neurol 2020. 20. Deeley Q. Hypnosis as therapy for functional neurologic disorders. Handb Clin Neurol 2016;139:585-595.

21. Terhune DB, Cardeña E. Dissociative subtypes in posttraumatic stress disorders and hypnosis: Neurocognitive parallels and clinical implications. Current Directions in Psychological Science $2015 ; 24: 452-457$.

22. Brown RJ, Reuber M. Psychological and psychiatric aspects of psychogenic non-epileptic seizures (PNES): A systematic review. Clin Psychol Rev 2016;45:157-182.

23. Holman L, Head ML, Lanfear R, Jennions MD. Evidence of experimental bias in the life sciences: Why we need blind data recording. PLoS Biol 2015;13:e1002190.

24. Terhune DB, Cleeremans A, Raz A, Lynn SJ. Hypnosis and top-down regulation of consciousness. Neurosci Biobehav Rev 2017;81:59-74.

25. Gudjonsson GH. Interrogative suggestibility and compliance. In: Ridley AM, Gabbert F, La Rooy DJ, eds. Suggestibility in legal contexts: Psychological research and forensic implications. Chichester, UK: Wiley-Blackwell, 2013: 45-61.

26. Thompson T, Terhune DB, Oram C, et al. The effectiveness of hypnosis for pain relief: A systematic review and meta-analysis of 85 controlled experimental trials. Neurosci Biobehav Rev 2019;99:298-310.

27. Hasselblad V, Hedges LV. Meta-analysis of screening and diagnostic tests. Psychol Bull $1995 ; 117: 167-178$. 
28. Egger M, Davey Smith G, Schneider M, Minder C. Bias in meta-analysis detected by a simple, graphical test. BMJ 1997;315:629-634.

29. Duval S, Tweedie R. Trim and fill: A simple funnel-plot-based method of testing and adjusting for publication bias in meta-analysis. Biometrics 2000;56:455-463.

30. Viechtbauer W, Cheung MW. Outlier and influence diagnostics for meta-analysis. Res Synth Methods 2010;1:112-125.

31. Higgins JPT, Green S. Cochrane handbook for systematic reviews of interventions. West Sussex: Wiley, 2008.

32. Yard SS, DuHamel KN, Galynker, II. Hypnotizability as a potential risk factor for posttraumatic stress: a review of quantitative studies. The International journal of clinical and experimental hypnosis 2008;56:334-356.

33. Martin JR, Pacherie E. Alterations of agency in hypnosis: A new predictive coding model. Psychol Rev 2019;126:133-152.

34. Roelofs K, Hoogduin KA, Keijsers GP, Naring GW, Moene FC, Sandijck P. Hypnotic susceptibility in patients with conversion disorder. J Abnorm Psychol 2002;111:390-395.

35. Khan AY, Baade L, Ablah E, McNerney V, Golewale MH, Liow K. Can hypnosis differentiate epileptic from nonepileptic events in the video/EEG monitoring unit? Data from a pilot study. Epilepsy Behav 2009;15:314-317.

36. Chabrol H, Peresson G, Clanet M. Lack of specificity of the traditional criteria for conversion disorders. Eur Psychiatry 1995;10:317-319.

37. Constant EL, Adam S, Gillain B, Lambert M, Masquelier E, Seron X. Cognitive deficits in patients with chronic fatigue syndrome compared to those with major depressive disorder and healthy controls. Clin Neurol Neurosurg 2011;113:295-302.

38. Wickramasekera IE. Somatization: Concepts, data, and predictions from the high risk model of threat perception. J Nerv Ment Dis 1995;183:15-23.

39. Frischholz EJ, Lipman LS, Braun BG, Sachs RG. Psychopathology, hypnotizability, and dissociation. Am J Psychiatry 1992;149:1521-1525.

40. Pettinati HM, Kogan LG, Evans FJ, Wade JH, Horne RL, Staats JM. Hypnotizability of psychiatric inpatients according to two different scales. Am J Psychiatry 1990;147:69-75. 
41. Holmes EA, Brown RJ, Mansell W, et al. Are there two qualitatively distinct forms of dissociation? A review and some clinical implications. Clin Psychol Rev 2005;25:1-23.

42. Kuyk J, Jacobs LD, Spinhoven P, van Dyck R. Use of a dissociation questionnaire and a hypnotizability scale in a population with pseudo- and epileptic seizures. Epilepsia 1995;36:S173.

43. Jankovic J. Diagnosis and treatment of psychogenic parkinsonism. J Neurol Neurosurg Psychiatry 2011;82:1300-1303.

44. Baizabal-Carvallo JF, Jankovic J. Examiner manoeuvres 'sensory tricks' in functional (psychogenic) movement disorders. J Neurol Neurosurg Psychiatry 2017;88:453-455.

45. Monday K, Jankovic J. Psychogenic myoclonus. Neurology 1993;43:349-352.

46. Edwards MJ, Bhatia KP, Cordivari C. Immediate response to botulinum toxin injections in patients with fixed dystonia. Mov Disord 2011;26:917-918.

47. Demartini B, Ricciardi L, Parees I, Ganos C, Bhatia KP, Edwards MJ. A positive diagnosis of functional (psychogenic) tics. Eur J Neurol 2015;22:527-e536.

48. Liu GT, Volpe NJ, Galetta SL. Functional (nonorganic) visual loss. In: Liu GT, Volpe NJ, Galetta SL, eds. Liu, Volpe, and Galetta's Neuro-Ophthalmology: Diagnosis and Management (3rd Ed): Elsevier, 2019: 379-393.

49. Kutlubaev MA, Xu Y, Hackett ML, Stone J. Dual diagnosis of epilepsy and psychogenic nonepileptic seizures: Systematic review and meta-analysis of frequency, correlates, and outcomes. Epilepsy Behav 2018;89:70-78.

50. Gogia B, Rai PK, Matthys SA, et al. Use of noninvasive induction techniques in the diagnosis of PNES. Epilepsy Behav 2019;99:106491.

51. Gambini O, Demartini B, Chiesa V, Turner K, Barbieri V, Canevini MP. Long-term outcome of psychogenic nonepileptic seizures: The role of induction by suggestion. Epilepsy Behav 2014;41:140-143. 52. Moene FC, Spinhoven P, Hoogduin KA, van Dyck R. A randomized controlled clinical trial of a hypnosis-based treatment for patients with conversion disorder, motor type. The International journal of clinical and experimental hypnosis 2003;51:29-50.

53. Popkirov S, Jungilligens J, Gronheit W, Wellmer J. Diagnosing psychogenic nonepileptic seizures: Video-EEG monitoring, suggestive seizure induction and diagnostic certainty. Epilepsy Behav 2017;73:5458. 


\section{Online supplemental content 1}

\section{Suggestibility in functional neurological disorder: A meta-analysis}

Lillian Wieder ${ }^{1}$, Richard J. Brown ${ }^{2,3}$, Che Ofuasia $^{1}$, Trevor Thompson ${ }^{4}$, \& Devin B. Terhune ${ }^{1}$

${ }^{1}$ Department of Psychology, Goldsmiths, University of London, London, UK

${ }^{2}$ School of Health Sciences, University of Manchester, Manchester, UK

${ }^{3}$ Complex Trauma and Resilience Research Unit, Greater Manchester Mental Health NHS Foundation Trust, Manchester Academic Health Sciences Centre, Manchester, UK

${ }^{4}$ School of Human Sciences, University of Greenwich, London, UK

\section{Correspondence address:}

Devin B. Terhune

Department of Psychology

Goldsmiths, University of London

8 Lewisham Way

New Cross, London, UK SE14 6NW

d.terhune@gold.ac.uk 


\section{Meta-analysis search terms}

The following search terms were used in the four databases (see main text): (hypno* OR suggestion OR suggestibility OR induc* OR placebo OR provocation) AND (psychogenic OR hysteri* OR somatis* OR somatiz* OR pseudo-neuro* OR "pseudo neuro*" OR pseudosens* OR "pseudoparalysis" OR "pseudo paralysis" OR pseudoseizure OR pseudo-seizure OR "pseudo-tumor" OR "functional neurological" OR "conversion disorder" OR non-epileptic OR nonepileptic OR PNES OR briquet* OR dissociative OR "medically unexplained")

\section{Methodological quality}

Endorsement of criteria $(1=$ criteria met, $0=$ criteria not met $)$

1: Was there a clear specification of study objectives?;

2: Was it clearly described where participants were drawn from (e.g. University etc.)?;

3: Was it clearly described how participants were recruited (e.g. advertisement, course credits, volunteers, etc.)?;

4: Was there a clear description of the inclusion and exclusion criteria?;

5: Was the experimenter blind to group?;

6: Were the diagnosis procedures and criteria clearly described?;

7: Was a rigorous suggestibility scale used?; [Standardized only]

8: Was the procedure and scale administration described in adequate detail?; [Standardized only]

9: Was a measure included to correct for compliance?; [Standardized only]

10: Was the symptom induction protocol clearly described?; [Symptom only]

11: Comparability of patients and controls;

12: Were relevant participant characteristics adequately described (age, sex etc.)?;

13: Were complete outcome data (i.e., $M$ s and $S D$ s) available (e.g. reported in article or given via response to data request)?

\section{Included studies}

Arain, A. M., Arbogast, P. G., \& Abou-Khalil, B. W. (2009). Utility of daily supervised hyperventilation during long-term video-EEG monitoring. Journal of Clinical Neurophysiology: Official Publication of the American Electroencephalographic Society, 26(1), 17-20. https://doi.org/10.1097/WNP.0b013e3181969032

Barry, J. J., Atzman, O., \& Morrell, M. J. (2000). Discriminating between epileptic and nonepileptic events: The utility of hypnotic seizure induction. Epilepsia, 41(1), 81-84. https://doi.org/10.1111/j.15281157.2000.tb01509.x

Bliss, E. L. (1984). Hysteria and hypnosis. Journal of Nervous and Mental Disease, 172(4), 203-206. https://doi.org/10.1097/00005053-198404000-00003

Brown, R. J., Schrag, A., Krishnamoorthy, E., \& Trimble, M. R. (2008). Are patients with somatization disorder highly suggestible? Acta Psychiatrica Scandinavica, 117(3), 232-235. https://doi.org/10.1111/j.1600-0447.2007.01127.x

Chabrol, H., Peresson, G., \& Clanet, M. (1995). Lack of specificity of the traditional criteria for conversion disorders. European Psychiatry: The Journal of the Association of European Psychiatrists, 10(6), 317319. https://doi.org/10.1016/0924-9338(96)80314-2

Chen-Block, S., Abou-Khalil, B. W., Arain, A., Haas, K. F., Lagrange, A. H., Gallagher, M. J., Azar, N. J., Singh, P., \& Sonmezturk, H. H. (2016). Video-EEG results and clinical characteristics in patients with psychogenic nonepileptic spells: The effect of a coexistent epilepsy. Epilepsy \& Behavior: E\&B, 62, 6265. https://doi.org/10.1016/j.yebeh.2016.06.018

Goldstein, L. H., Drew, C., Mellers, J., Mitchell-O’Malley, S., \& Oakley, D. A. (2000). Dissociation, hypnotizability, coping styles and health locus of control: Characteristics of pseudoseizure patients. Seizure, 9(5), 314-322. https://doi.org/10.1053/seiz.2000.0421

Goyal, G., Kalita, J., \& Misra, U. K. (2014). Utility of different seizure induction protocols in psychogenic nonepileptic seizures. Epilepsy Research, 108(6), 1120-1127.

https://doi.org/10.1016/j.eplepsyres.2014.02.015 
Kandler, R., Lawrence, S., Pang, C., Lai, M., \& Whitehead, K. (2018). Optimising the use of EEG in nonepileptic attack disorder: Results of a UK national service evaluation. Seizure, 55, 57-65. https://doi.org/10.1016/j.seizure.2018.01.005

Kenney, C., Diamond, A., Mejia, N., Davidson, A., Hunter, C., \& Jankovic, J. (2007). Distinguishing psychogenic and essential tremor. Journal of the Neurological Sciences, 263(1-2), 94-99. https://doi.org/10.1016/j.jns.2007.06.008

Khan, A. Y., Baade, L., Ablah, E., McNerney, V., Golewale, M. H., \& Liow, K. (2009). Can hypnosis differentiate epileptic from nonepileptic events in the video/EEG monitoring unit? Data from a pilot study. Epilepsy \& Behavior: E\&B, 15(3), 314-317. https://doi.org/10.1016/j.yebeh.2009.04.004

Kuyk, J., Spinhoven, P., \& van Dyck, R. (1999). Hypnotic recall: A positive criterion in the differential diagnosis between epileptic and pseudoepileptic seizures. Epilepsia, 40(4), 485-491. https://doi.org/10.1111/j.1528-1157.1999.tb00745.x

Kuyk, Jarl, Jacobs, L. D., Aldenkamp, A. P., Meinardi, H., Spinhoven, P., \& Van Dycki, R. (1995). Pseudoepileptic seizures: Hypnosis as a diagnostic tool. Seizure, 4(2), 123-128. https://doi.org/10.1016/S10591311(95)80091-3

Lancman, M. E., Asconapé, J. J., Craven, W. J., Howard, G., \& Penry, J. K. (1994). Predictive value of induction of psychogenic seizures by suggestion. Annals of Neurology, 35(3), 359-361. https://doi.org/10.1002/ana.410350319

Litwin, R., \& Cardeña, E. (2000). Demographic and seizure variables, but not hypnotizability or dissociation, differentiated psychogenic from organic seizures. Journal of Trauma \& Dissociation, 1(4), 99-122. https://doi.org/10.1300/J229v01n04_06

Martínez-Taboas, A. (2002). The role of hypnosis in the detection of psychogenic seizures. The American Journal of Clinical Hypnosis, 45(1), 11-20. https://doi.org/10.1080/00029157.2002.10403493

Moene, F. C., Spinhoven, P., Hoogduin, K., Sandyck, P., \& Roelofs, K. (2001). Hypnotizability, dissociation and trauma in patients with a conversion disorder: An exploratory study. Clinical Psychology \& Psychotherapy, 8(6), 400-410. https://doi.org/10.1002/cpp.293

Roelofs, K., Hoogduin, K. A. L., Keijsers, G. P. J., Näring, G. W. B., Moene, F. C., \& Sandijck, P. (2002). Hypnotic susceptibility in patients with conversion disorder. Journal of Abnormal Psychology, 111(2), 390-395. https://doi.org/10.1037/0021-843X.111.2.390

Spinhoven, P., Van Dyck, R., Hoogduin, K., \& Schaap, C. (1991). Differences in hypnotisability of Dutch psychiatric outpatients according to two different scales. Australian Journal of Clinical \& Experimental Hypnosis, 19(2), 107-116.

Walczak, T. S., Williams, D. T., \& Berten, W. (1994). Utility and reliability of placebo infusion in the evaluation of patients with seizures. Neurology, 44(3 Pt 1), 394-399.

https://doi.org/10.1212/wnl.44.3_part_1.394 
Supplementary Table 1. Endorsement of methodological criteria for standardized suggestibility studies (1= criteria met, $0=$ criteria not met)

\begin{tabular}{|c|c|c|c|c|c|c|c|c|c|c|c|c|c|}
\hline \multirow[t]{2}{*}{ Source } & \multicolumn{12}{|c|}{ Question } & \multirow[t]{2}{*}{ Total } \\
\hline & 1 & 2 & 3 & 4 & 5 & 6 & 7 & 8 & 9 & 11 & 12 & 13 & \\
\hline Khan et al., 2009 & 1 & 1 & 1 & 1 & 0 & 1 & 1 & 0 & 0 & 0 & 0 & 1 & 7 \\
\hline Brown et al., 2008 & 1 & 1 & 1 & 1 & 0 & 1 & 1 & 1 & 1 & 0 & 1 & 1 & 10 \\
\hline Roelofs et al., 2002 & 1 & 1 & 1 & 1 & 1 & 1 & 1 & 1 & 0 & 1 & 1 & 1 & 11 \\
\hline Litwin and Cardeña, 2001 & 1 & 1 & 1 & 1 & 1 & 1 & 1 & 1 & 0 & 0 & 1 & 1 & 10 \\
\hline $\begin{array}{l}\text { Moene et al., } 2001 \text { / } \\
\text { Spinhoven et al. 1991* }\end{array}$ & 1 & 1 & 1 & 1 & 0 & 1 & 1 & 1 & 0 & 0 & 0 & 1 & 8 \\
\hline Barry et al., 2000 & 1 & 1 & 1 & 1 & 0 & 1 & 1 & 0 & 0 & 0 & 0 & 1 & 7 \\
\hline Goldstein et al., 2000 & 1 & 1 & 1 & 1 & 0 & 1 & 1 & 1 & 0 & 0 & 1 & 1 & 9 \\
\hline Kuyk et al., 1999 & 1 & 1 & 1 & 1 & 1 & 0 & 1 & 1 & 0 & 0 & 1 & 1 & 9 \\
\hline Kuyk et al., 1995 & 1 & 1 & 1 & 1 & 1 & 1 & 1 & 1 & 0 & 0 & 1 & 1 & 10 \\
\hline Bliss, 1984a & 0 & 1 & 1 & 0 & 0 & 0 & 1 & 0 & 0 & 0 & 0 & 1 & 4 \\
\hline Bliss, 1984b & 1 & 1 & 1 & 0 & 0 & 0 & 1 & 0 & 0 & 0 & 0 & 1 & 5 \\
\hline
\end{tabular}

* = Control data were drawn from Spinhoven et al. (1991).

Supplementary Table 2. Endorsement of methodological criteria for symptom suggestibility studies (1= criteria met, $0=$ criteria not met)

\begin{tabular}{|c|c|c|c|c|c|c|c|c|c|c|c|}
\hline \multirow[t]{2}{*}{ Source } & \multicolumn{10}{|c|}{ Question } & \multirow[t]{2}{*}{ Total } \\
\hline & 1 & 2 & 3 & 4 & 5 & 6 & 10 & 11 & 12 & 13 & \\
\hline Kandler et al., 2018 & 1 & 1 & 1 & 1 & 0 & 0 & 0 & 0 & 0 & 1 & 5 \\
\hline $\begin{array}{l}\text { Chen-Block et al., } \\
2016\end{array}$ & 1 & 1 & 1 & 1 & 0 & 1 & 0 & 0 & 0 & 1 & 6 \\
\hline Goyal et al., 2014 & 1 & 1 & 1 & 1 & 0 & 1 & 1 & 0 & 1 & 1 & 8 \\
\hline Arain et al., 2009 & 1 & 1 & 1 & 1 & 0 & 0 & 1 & 0 & 0 & 1 & 6 \\
\hline Khan et al., 2009 & 1 & 1 & 1 & 1 & 0 & 1 & 1 & 0 & 0 & 1 & 7 \\
\hline Kenney et al., 2007 & 1 & 1 & 1 & 1 & 1 & 1 & 1 & 0 & 0 & 1 & 8 \\
\hline Martinez-Taboas, 2002 & 1 & 1 & 1 & 0 & 0 & 1 & 1 & 1 & 1 & 1 & 8 \\
\hline Barry et al., 2000 & 1 & 1 & 1 & 1 & 0 & 1 & 1 & 0 & 0 & 1 & 7 \\
\hline Chabrol et al, 1995 & 1 & 1 & 1 & 1 & 0 & 0 & 0 & 0 & 0 & 1 & 5 \\
\hline Lancman et al., 1994 & 1 & 0 & 0 & 1 & 0 & 1 & 1 & 1 & 1 & 1 & 7 \\
\hline Walczak et al., 1994 & 1 & 1 & 1 & 0 & 0 & 1 & 1 & 0 & 1 & 1 & 7 \\
\hline
\end{tabular}

